\title{
MHD turbulence and heating of the open field-line solar corona
}

\author{
W. H. Matthaeus ${ }^{1}$, D. J. Mullan ${ }^{1}$, P. Dmitruk ${ }^{1}$, L. Milano ${ }^{1}$, and S. Oughton ${ }^{2}$ \\ ${ }^{1}$ University of Delaware, Newark DE 19716, USA \\ ${ }^{2}$ University of Waikato, Hamilton, New Zealand
}

Received: 8 March 2002 - Revised: 23 May 2002 - Accepted: 24 May 2002

\begin{abstract}
This paper discusses the possibility that heating of the solar corona in open field-line regions emanating from coronal holes is due to a nonlinear cascade, driven by low-frequency or quasi-static magnetohydrodynamic fluctuations. Reflection from coronal inhomogeneities plays an important role in sustaining the cascade. Physical and observational constraints are discussed. Kinetic processes that convert cascaded energy into heat must occur in regions of turbulent small-scale reconnection, and may be similar in some respects to ion heating due to intense electron beams observed in the aurora.
\end{abstract}

\section{Introduction}

There are long standing puzzles concerning the existence of the solar corona, prominent among which are the related issues of heating the corona and accelerating the solar wind (Ulmschneider, 1991). The issues became more focused and in some ways more puzzling when, first, Ulysses revealed the smooth, steady properties of the polar wind, and then, in rapid succession instruments aboard Spartan sounding rockets, and the Solar and Heliospheric Observatory (SOHO) spacecraft, as well as ground-based radio astronomy observations (Kohl et al., 1995, 1997; Grall et al., 1996) indicated that at low altitudes (within several solar radii $\left(R_{\odot}\right)$ above the photosphere) the corona is hotter and the wind faster than had been believed earlier. To address the central underlying physics, a new generation of coronal heating models has arisen, emphasizing the need to understand heating in open field-line regions from which the fast polar wind emerges. Magnetic fluctuations originating in the photosphere remain an important energy conduit, but we now also know the open field-line corona must be highly dynamic within 1 solar radius of the photosphere, with proton temperatures reaching temperatures in excess of 1 million $\mathrm{K}$, minor ions hotter still,

Correspondence to: W. H. Matthaeus

(yswhm@bartol.udel.edu) and the wind accelerated to several hundred $\mathrm{km} / \mathrm{s}$. Moreover, all these must happen within tight constraints of time and distance.

In this brief review we attempt to provide an observationally based overview of open field-line coronal heating. We recognize at the onset that a satisfactory mechanism involves energy transport through several distinct regions: the photosphere, the chromosphere, a possibly poorly defined transition region, and then the inhomogeneous corona. By comparing and discussing these coupled layers, a useful perspective on the requirements for a coronal heating mechanism can be achieved.

The particular mechanism we focus upon is one in which the coronal hole plasma is heated due to a transverse turbulent magnetohydrodynamic (MHD) cascade, driven by mixed polarization low-frequency Alfvénic fluctuations. The process is triggered by fluctuations with periods of 100$1000 \mathrm{~s}$, or longer, impinging from beneath the corona, and catalyzed by reflections from the Alfvén speed gradients. The basic geometry is illustrated in Fig. 1. We offer this as an alternative approach to more familiar mechanisms (e.g. Axford and McKenzie, 1997) that rely upon copious high frequency waves with periods of $0.001-0.01 \mathrm{~s}$, that directly cyclotron damp. Finally we offer some thoughts on possible kinetic mechanisms that might convert MHD energy into heat, driven by the strong cascade to highly transverse (oblique) wavenumbers that emerges naturally in a theory of a turbulent corona driven by low-frequency or quasi-static fluctuations.

The photosphere of the Sun separates regions in which very different physical processes control energy transport. Below the photosphere, thermal transport is the controlling process, whereas above the photosphere mechanical energy dominates. Still higher up in the atmosphere, the corona is dominated by magnetic energy, and transport processes become magnetohydrodynamic (MHD) in nature. An explanation of coronal heating must take all of these processes into account and provide a description of the mechanisms by which the energy is being transferred (i) through the different 
regions, and (ii) across spatial scales to reach the small scales where it can be efficiently dissipated. Ultimately, much of the energy returns to thermal motions in the corona, producing the solar wind and establishing the baseline conditions for the entire heliosphere.

\section{The Sun's outer atmosphere: mechanical energy at work}

At heights $z \approx 500 \mathrm{~km}$ above the photosphere, the temperature begins to increase outward, forming the chromosphere. The chromospheric temperature rise indicates that mechanical energy is being continually deposited there. The flux of mechanical energy that is deposited in the quiet or active chromosphere is $(0.4-2) \times 10^{7} \mathrm{ergs} / \mathrm{cm}^{2} / \mathrm{s}$ (Withbroe and Noyes, 1977).

At $z \geq 2 \mathrm{Mm}$, temperatures rise rapidly to $\geq 10^{6} \mathrm{~K}$. The million-degree plasma (the "solar corona") is hottest and densest in "active regions", where closed magnetic loops exist. In solar X-ray images, closed loops are bright, while "coronal holes" (open field regions) are dark. In the so-called "quiet Sun", there exists a diffuse featureless glow of X-rays. The flux of mechanical energy required for the quiet corona is $3 \times 10^{5} \mathrm{ergs} / \mathrm{cm}^{2} / \mathrm{s}$ (Withbroe and Noyes, 1977).

Interest in coronal heating is not confined only to solar physics. Other stars also emit strongly in X-rays, especially if the star is "active", i.e. has short-lived releases of magnetic energy ("flares") (e.g. Mullan and Fleming, 1996).

In our discussion of coronal physics, the density contrast between photosphere and corona $n_{\mathrm{ph}} / n_{\mathrm{c}}$ plays an important role. In the Sun, this contrast is of order $10^{8}$ (Vernazza et al., 1981).

\section{Chromosphere versus corona: hydrodynamics versus MHD}

Our aim is to model coronal heating. To do this, we must consider the generation, propagation, and dissipation of mechanical energy in the conditions which prevail in the upper layers of the Sun and solar-like stars. Turbulence and nonlinearities play key roles in such a model.

Although our ultimate focus is the corona, where magnetic effects are key, it is instructive first to consider the heating of the chromosphere. Chromospheric heating can be modeled almost entirely in the context of hydrodynamics. Because there are fewer free parameters in the theory of acoustic wave propagation, it is easier to identify the requisite components of successful models of chromospheric heating.

3.1 Chromospheric heating: acoustic emission from convective turbulence

The quiet solar chromosphere is heated by acoustic fluctuations which are generated in the convective turbulence that exists in (and below) the solar photosphere. The random nature of solar convection means that acoustic fluctuations are created with a range of amplitudes $\delta v$ over a spectrum of frequencies $v$. This spectrum is determined by the spatial and temporal spectra of the turbulent motions (Musielak et al., 1994). Empirically, the spatial spectrum of solar convection peaks on scales of 1-2 Mm (Namba and Diemel, 1969). The temporal spectrum of solar convection peaks at long periods ( $P=700-800 \mathrm{~s}$ : the typical granule lifetime), with rms velocity amplitudes of $0.5-0.6 \mathrm{~km} / \mathrm{s}$. There is also a peak at 200-250 s (associated with $p$-modes), with rms amplitudes of $0.8-0.9 \mathrm{~km} / \mathrm{s}$ (Ionson, 1984). For $P \leq 100 \mathrm{~s}$, the power spectrum $S(v) \sim v^{-2}$ (Milano et al., 1997), which is a rather steep fall-off.

3.2 Chromospheric heating: the upward flux of propagating acoustic waves

Chromospheric heating relies on waves with $P$ shorter than the acoustic cut-off $P_{\text {cutoff }}=4 \pi c_{\mathrm{s}} / \gamma g=200-300 \mathrm{~s}$. Peak acoustic power occurs around $P_{\text {cutoff }} / 5$ (i.e., $40-60 \mathrm{~s}$ in the Sun), with an exponentially steep fall-off in power at shorter periods (Ulmschneider, 1991). Integrating over this spectrum, the energy flux $F_{\text {ac }}$ of acoustic waves available for chromospheric heating is $5 \times 10^{7} \mathrm{ergs} / \mathrm{cm}^{2} / \mathrm{s}$ (Musielak et al., 1994).

Although there are uncertainties in this estimate, it is gratifying that $F_{\text {ac }}$ suffices to satisfy the $(0.4-2) \times 10^{7} \mathrm{ergs} / \mathrm{cm}^{2} / \mathrm{s}$ energy budget of the solar chromosphere (Withbroe and Noyes, 1977). Moreover, noting that $F_{\mathrm{ac}} \approx \rho(\delta v)^{2} c_{\mathrm{s}}$, we find that acoustic waves in the photosphere are launched upwards with Mach numbers $\delta v / c_{\mathrm{s}}$ of about 0.02 .

\subsection{Chromospheric heating: nonlinear acoustics}

Nonlinear development of acoustic waves depends on the ambient medium and on the wave period. As regards the ambient medium, the solar atmosphere above the photosphere has a steeply declining density profile: the density scaleheight, $H_{d}=R_{g} T / \mu g$, is only $100-110 \mathrm{~km}$. Acoustic waves injected at the photosphere steepen rapidly as they go upward: $\delta v \sim \rho^{-0.5} \sim \exp \left(z / 2 H_{d}\right)$, in order to conserve energy. As a result, waves in the photosphere with amplitude $\delta v / c_{\mathrm{s}} \approx 0.02$ grow in amplitude to $(0.2-0.3) c_{\mathrm{s}}$ at $z=500 \mathrm{~km}$. Nonlinear steepening leads to shocks, which dissipate at a volumetric heating rate of $\dot{E}_{\text {mech }} \approx F_{\mathrm{ac}} / H_{d}$. It is this dissipation that causes the chromospheric temperature rise.

As regards the wave period, long-period waves (with periods close to $P_{\text {cutoff }} \approx 300 \mathrm{~s}$ ) dissipate at greater heights in the atmosphere than short-period waves (Stein and Schwartz, 1973). As a result, waves with $P \approx 40-60$ s dominate in heating the low chromosphere, while waves with $P \approx 300 \mathrm{~s}$ heat the upper chromosphere.

Hydrodynamic theory provides a satisfactory quantitative explanation for the heating of the chromosphere in the quiet Sun (Ulmschneider, 1991). In fact, when favorable observing conditions allow one to specify the amplitude and temporal behavior of photospheric motions in detail, hydrody- 
namical modeling has achieved remarkable success in reproducing the time-dependent phenomena in the overlying quiet chromosphere (Carlsson and Stein, 1997). Acoustic emission also accounts well for a "basal" component of chromospheric emission in other cool stars (Mullan and Cheng, 1993; Buchholz et al., 1998).

\section{Coronal heating: the necessary ingredients}

Although acoustic heating may be capable of heating coronae in certain $\mathrm{M}$ dwarfs, where the magnetic activity level is very low (Mullan and Cheng, 1994), acoustic waves are simply not efficient in heating the corona in the Sun or solar-like stars (Hammer and Ulmschneider, 1991). A magnetic process is essential for solar coronal heating. Does this magnetic process necessarily involve waves in the corona? Strictly speaking, no. A "nanoflare" model (Parker, 1988) involving reconnection events may explain heating in closed magnetic loops. But nanoflares cannot explain coronal heating in open field regions. Moreover, the distinction between lowfrequency wave driving and quasi-static driving (familiar in loop heating models - see below) becomes blurred when the implied wave period exceeds the time to propagate across the system. As an example, taking the system size to be $\sim R_{\odot}$ and the Alfvén speed to be $1000-2500 \mathrm{~km} / \mathrm{s}$, the distinction more or less disappears for driving waves with periods longer than 300-700 s.

However, the combination of magnetism and convection in the Sun has the effect that MHD waves are present in the solar corona as surely as acoustic waves are present in the chromosphere. Thus, it is natural to use MHD waves to heat the corona. In addition, the corona presumably has a low plasma beta and a strong large-scale magnetic field. This favors the kinetic damping of magnetoacoustic waves, and the predominance of nearly-incompressible Alfvénic fluctuations (e.g. Barnes, 1979). Consequently it is reasonable to begin turbulent heating models with a locally incompressible description based upon Alfvén mode fluctuations.

To obtain a successful coronal model, the hydrodynamic discussion of the chromosphere given above indicates that knowledge of the following items will be essential: (1) initial wave amplitudes, (2) the wave flux which reaches the corona, (3) the spectrum of the waves, both temporal and spatial, and (4) the properties of the ambient medium.

\subsection{MHD wave amplitudes}

Magnetic flux tubes which permeate the solar corona are rooted in the photosphere, where the plasma $\beta$ parameter $\left(\beta=p_{\text {gas }} / p_{\text {mag }}\right)$ is of order unity in magnetically active regions. In the photosphere, the convective motions jostle the footpoints of magnetic flux tubes such that the velocity amplitudes $\delta v_{\text {MHD }}$ of MHD waves in the photosphere are comparable to horizontal velocities there, i.e. $0.5-5 \mathrm{~km} / \mathrm{s}$ (Berger and Title, 1996). The associated flux of MHD waves, $F_{\text {MHD, }}$ is of order $\rho\left(\delta v_{\mathrm{MHD}}\right)^{2} V_{A}$, where $V_{A}$ is the Alfvén speed.

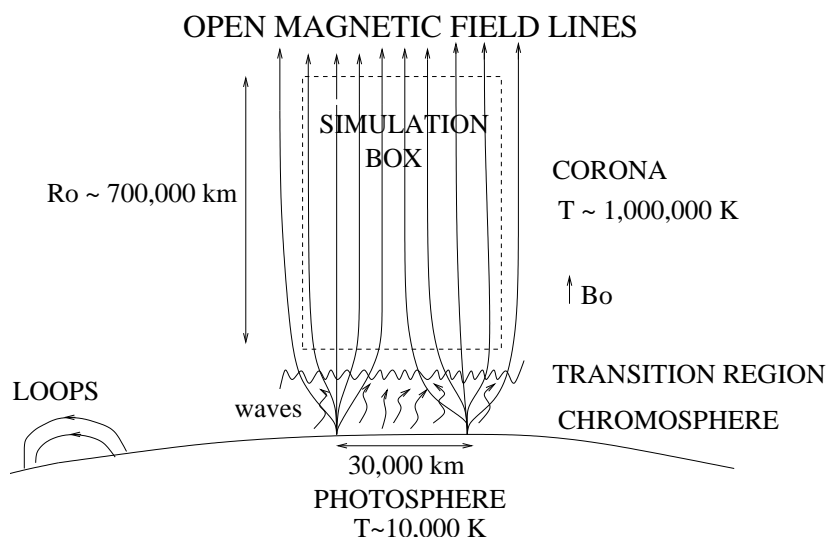

Fig. 1. Sketch of the corona and surrounding regions, suggesting a flow of fluctuation energy that drives a turbulent heating process. Temperatures indicated are approximate proton temperatures.

Stratification causes $\delta v_{\mathrm{MHD}}$ to increase upwards as $\rho^{-0.25}$ if $F_{\mathrm{MHD}}$ and $B$ are constant with altitude. With a density contrast of $10^{8}$ between photosphere and corona, $\delta v_{\mathrm{MHD}}$ at the base of the corona should exceed the photospheric amplitude by a factor of 100 . This would lead to coronal wave amplitudes $\delta v_{\text {cor }}$ of $50-500 \mathrm{~km} / \mathrm{s}$. However, this is an extreme upper limit: neither the field strength nor $F_{\mathrm{MHD}}$ remain constant with altitude in the Sun. To obtain more realistic values of wave amplitudes at the base of the corona, we need to consider the problem of wave transmission through a density gradient.

\subsection{Transmission of waves into the corona}

The fraction $\phi_{\text {Alf }}$ of Alfvén waves launched from the photosphere that penetrate into the corona has been estimated by Leer et al. (1982): for a certain wave period, they find $\phi_{\text {Alf }} \approx$ 0.006 . This is so small that Leer et al. (1982) considered it a fatal objection to models where coronal heating relies on MHD waves from the photosphere. However, more recent data (Berger and Title, 1996) indicate that "the power input into the corona is an order of magnitude larger than previously calculated". As a result, the small value of $\phi_{\text {Alf }}$ is not the fatal objection that it appeared to Leer et al. (1982).

Using these estimates of $\phi_{\text {Alf }}$, the amplitudes $\delta v_{\text {cor }}$ that are expected to occur at the base of the corona are reduced below our estimates above by factors of $1 / \sqrt{\phi_{\text {Alf }}} \approx 13$. This leads to an upper limit on $\delta v_{\text {cor }}$ of about $40 \mathrm{~km} / \mathrm{s}$. Empirical estimates of spectral line broadening in the low corona do indeed indicate amplitudes of $20-50 \mathrm{~km} / \mathrm{s}$ (Hassler et al., 1990; Chae et al., 1998).

For consistency, we note that in order for a flux $F_{\text {cor }} \approx$ $3 \times 10^{5} \mathrm{ergs} / \mathrm{cm}^{2} / \mathrm{s}$ (Withbroe and Noyes, 1977) to reach the corona, the photosphere must launch a flux $F_{\mathrm{ph}}=$ $F_{\text {cor }} / \phi_{\text {Alf }} \approx 5 \times 10^{7} \mathrm{ergs} / \mathrm{cm}^{2} / \mathrm{s}$. With $\delta v=(0.5-5) \mathrm{km} / \mathrm{s}$ in the photosphere (Berger and Title, 1996), we find that $F_{\mathrm{ph}} \approx \rho(\delta v)^{2} V_{A}$ requires that $V_{A} \approx(0.007-0.7) \mathrm{km} / \mathrm{s}$. These values of $V_{A}$ correspond to field strengths of 1.4- 
140 Gauss in the photosphere. Fields with strengths in the lower end of this range are common even in the quietest regions of the Sun (Foukal, 1990). In active regions, mean field strengths are often as large as $140 \mathrm{G}$.

Thus, even though the density contrast between photosphere and corona presents a serious obstacle as regards transmission of MHD waves to the corona, conditions in the Sun are such that adequate MHD wave energy can be supplied from the photosphere to satisfy the coronal energy budget.

\subsection{The spectrum of MHD waves: temporal and spatial}

Since the MHD waves emerging from the photosphere are emitted in interactions between convection and magnetic field tubes, it seems likely that the wave spectrum will be dominated by the periods which are typical of convective motions. To the extent that this is valid, the MHD waves in the solar atmosphere will have a double-peaked power spectrum (Ionson, 1984), with the peaks at 700-800 s and 200-250 s. The power should fall off steeply towards shorter periods, perhaps as fast as $P^{2}$. Therefore, the MHD power at periods of (say) 0.01 or 0.001 seconds may be $10^{10}-10^{12}$ times smaller than at $1000 \mathrm{~s}$.

The spatial structure of MHD waves that are launched into the corona is determined by the spatial organization of fields in the photosphere. This organization is controlled by supergranules, where large-scale ( $L \approx 20-30 \mathrm{Mm}$ ) horizontal flows sweep magnetic field lines from the center of a supergranule cell towards the periphery. The accumulation of magnetic flux along the periphery of a supergranule cell gives rise to locally enhanced emission in a "network". Our model incorporates this spatial organization by launching waves into the lower boundary with 2D structure corresponding to supergranule dimensions. In simulations, our lower boundary is large enough to include, typically, four supergranules. The geometry we employ in our numerical modeling of this region is suggested in Fig. 1. We will now discuss the nature of turbulence driven by low-frequency waves in this representation of a section of the lower corona.

\subsection{Nonlinear wave interactions: counter-streaming waves}

In the corona, the vertical gradient of the Alfvén speed $V_{A}(z)$ causes MHD nonlinearities to develop. In the presence of a vertical gradient of $V_{A}$, upward propagating Alfvén waves are partially reflected (An et al., 1990). Reflection is essentially complete for Alfvén waves whose wavelengths $\lambda_{A}=$ $P_{A} V_{A}$ exceed the lengthscale $H_{A}=d z / d \ln V_{A}$ over which $V_{A}$ varies. ( $P_{A}$ is the wave period.)

Reflection creates a flux of downward propagating waves, and the counter-streaming of upward and downward waves gives rise to the nonlinear interactions that are at the heart of our model. These interactions result in a turbulent cascade of a particular kind: energy cascades towards small structures that are oriented mainly perpendicular to the mean field direction (Matthaeus et al., 1999; Leamon et al., 2000;
Oughton et al., 2001; Dmitruk et al., 2001). We illustrate this cascading process in Fig. 2, which shows cross sections from a three dimensional Reduced MHD (RMHD) simulation of this process. RMHD is appropriate to describe low frequency dynamics in the presence of a strong mean magnetic field, especially at low plasma beta (see Oughton et al., 2001, and references therein). The simulation generating the results shown is a three dimensional $(128 \times 128 \times 16$ resolution) RMHD Fourier-Chebyshev code with open boundaries. In the first panel, we show the wave flow structure injected at the coronal base, with several supergranule-like structures in evidence. In the second panel, we show the spatial structure in a horizontal cut through the computational domain at higher coronal altitude after some time has elapsed. The second panel indicates that spatial structure has developed on much smaller transverse scales than those which were injected at the lower boundary. This perpendicular cascade is the primary manifestation of the nonlinear interactions that occur as a result of counter-streaming waves (Oughton et al., 2001; Dmitruk et al., 2001).

We emphasize that the cascade which is at work in our model is essentially distinct from a certain type of cascade that is often assumed (without justification) in the literature, namely a cascade in the Alfvén wave spectrum towards higher frequencies in the direction parallel to the mean field. As far as we know, there are neither theoretical predictions nor simulation results which indicate that such a parallel cascade in frequency ever occurs. Instead, turbulence involves a cascade in wavenumber at essentially constant (low) frequency. An essential feature of our model is that the Alfvén waves propagate parallel to the field, whereas the turbulent cascade proceeds transverse to the field.

\subsection{Properties of the ambient medium: scale-height for re- flections}

Since reflection occurs on length-scales of order $H_{A}$, and since energy deposition occurs rapidly once counterstreaming waves are present, the length-scale for energy deposition $H_{E}$ is expected to be comparable to $H_{A}$ in our model. Let us estimate typical values of $H_{A}$ in coronal conditions.

As $V_{A} \sim B / \sqrt{\rho}$, we have $1 / H_{A}=1 / H_{B}+1 /\left(2 H_{d}\right)$. Here, $H_{B}$ is the field scale-height and $H_{d}$ is the density scaleheight.

It is straightforward to estimate $H_{d}$ in the low corona. Since the wind flow speeds there are small compared to the speed of sound, hydrostatic equilibrium (HSE) is a good approximation: $d p / d r=-\rho g$. In an isothermal corona, HSE leads to a density profile $\rho(r)=\rho_{0} \exp \left[\alpha\left(R_{\odot} / r-1\right)\right]$, where $\alpha=\mu G M_{\odot} / R_{g} T R_{\odot}$. (Here, $\mu$ is the mean molecular weight and $R_{g}$ is the gas constant.) For a fully ionized hydrogen plasma at $T=10^{6} \mathrm{~K}, \alpha \approx 12$. Therefore, the density in the low corona falls off with scale-height $H_{d} \approx R_{\odot} / \alpha \approx 50$ $60 \mathrm{Mm}$.

As regards the value of $H_{B}$ in the low corona, the answer is less definitive. There are no observations that allow field 


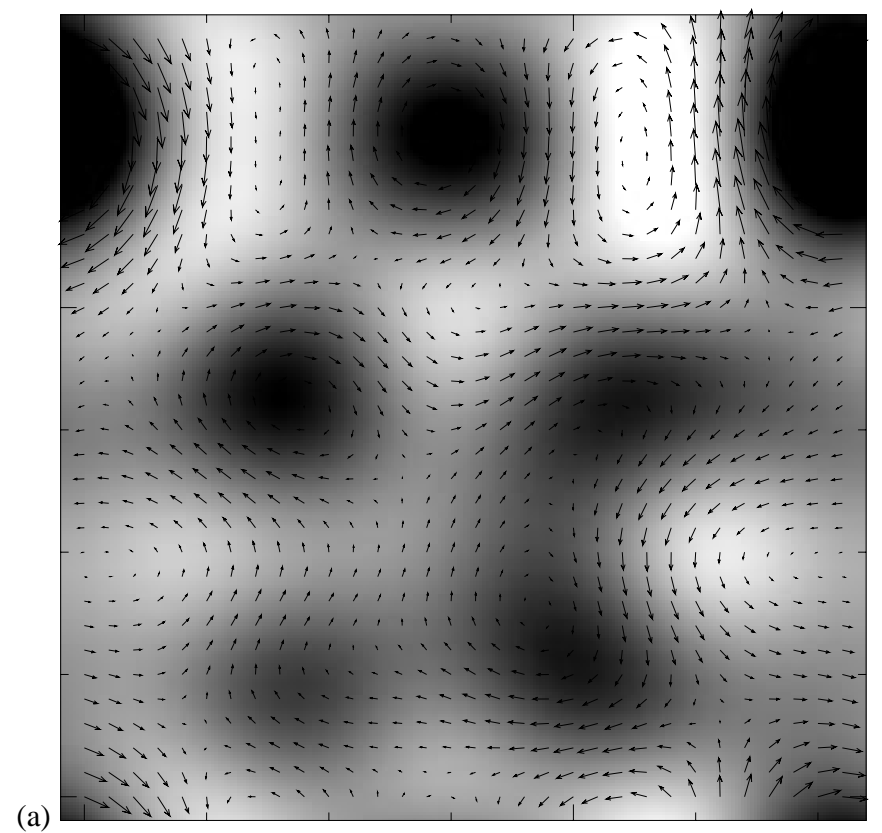

(a)

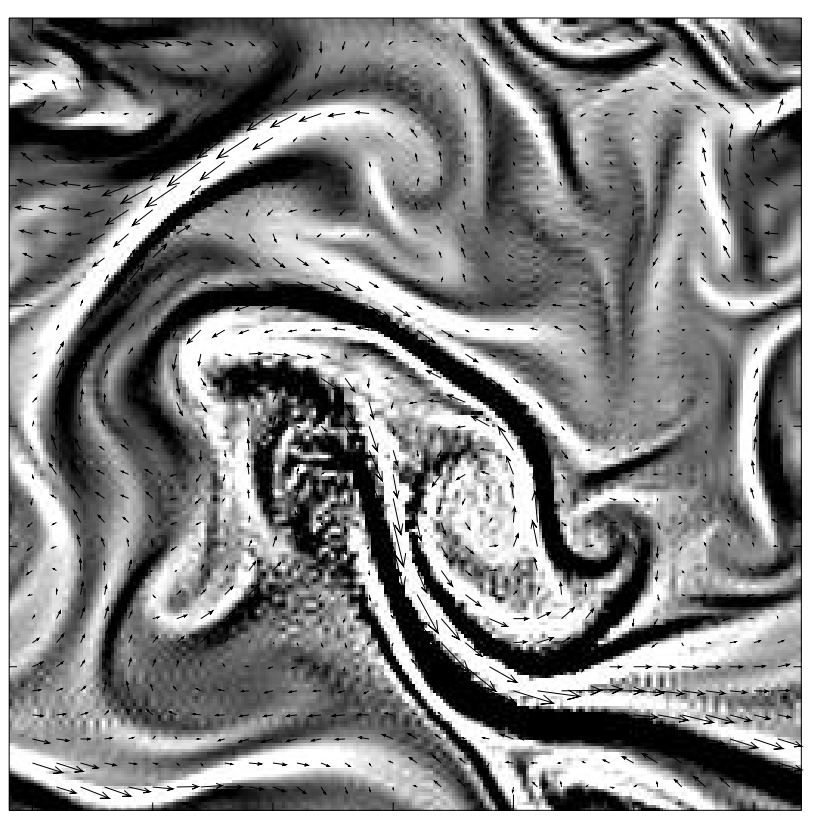

(b)

Fig. 2. Transverse sections of the turbulence in a Reduced MHD (RMHD) model driven by low-frequency fluctuations imposed at the bottom. The transverse box size depicted corresponds to $\approx$ $100000 \mathrm{~km}$ in the corona. The simulation extends one solar radius in the radial direction. Magnetic field-lines are shown as arrows; electric current density is shown as grayscale Very dark and very light corresponds, respectively, to intense positive or negative current density. (a) Section at the bottom, showing the transverse structure at the scale of the network $(30000 \mathrm{~km})$ imposed at the base. (b) Evolved transverse structure a fraction of a solar radius above the base. Turbulence leads to driven random current sheets and reconnection, resulting in strong deposition of heat in the lower part of the modeled corona.

strengths to be measured as a function of $z$ in the coronal portions of an open field region. Extrapolation of photo- spheric fields is required, and this inevitably requires certain assumptions. Assuming that the coronal fields are currentfree, Schrijver and Aschwanden (2001) have recently fitted calculated fields to a number of coronal loops observed by TRACE. They find that over solar active regions, $H_{B}$ (averaged over all active regions) is about $15 \mathrm{Mm}$.

\subsection{Energy deposition in the corona: length-scales}

Using the above values of $H_{B}$ and $H_{d}$, we see that $H_{A} \approx$ $15 \mathrm{Mm}$. In the context of our reflection scenario, this means that the energy deposition scale $H_{E}$ should also be of order $15 \mathrm{Mm}$, in these circumstances.

Is there any empirical support for this result? In coronal holes, there is no evidence to decide: the intensity of X-ray emission is too small to allow one to deduce $H_{E}$ in the low corona. However, in certain closed loops, $H_{E}$ can be estimated by modeling TRACE data. At first sight, it might appear that what happens in closed loops should have little relevance to our coronal heating model: after all, the latter assumes an open field. However, a loop which has a length $L$ that greatly exceeds $H_{E}$ can be considered from the point of view of energy deposition, as consisting of two "open field regions" at the loop footpoints, where the imposed photospheric velocity field implies a reflection condition at the footpoint planes (acting like "wall" boundaries). Therefore, the properties of loops with $L \geq H_{E}$ are not irrelevant to our discussion. In a sample of 41 such loops, Aschwanden et al. (2000) have determined $H_{E}=17 \pm 6 \mathrm{Mm}$.

This empirical result overlaps gratifyingly with the predictions $\left(H_{E}=15 \mathrm{Mm}\right)$ of our reflection scenario.

\subsection{Periods of waves that are efficiently reflected}

The minimum period that satisfies the reflection criterion is $P_{\min }=H_{A} / V_{A}$. With densities and field strengths appropriate to the base of a coronal hole, one finds $V_{A}=$ $2500 \mathrm{~km} / \mathrm{s}$ at the base of the corona (Axford and McKenzie, 1997). Then the upper limit on $P_{\min }$ is $\leq 10 \mathrm{~s}$. As a result, most of the waves emerging from the solar convection zone (with $P=10^{2}-10^{3} \mathrm{~s}$ ) are subject to efficient reflection.

\subsection{Heating in closed field line regions}

Several models for turbulent coronal heating in closed field line regions (or coronal loops) have been proposed to present. Turbulence models are part of a broader category of coronal heating models, that is, models based on quasi-static phtospheric mechanical stress on the coronal magnetic field, or DC models. Such a driving produces small scale magnetic structures (current sheets), capable of efficient Joule dissipation in the corona (Parker, 1972, 1983; Heyvaerts and Priest, 1983; van Ballegooijen, 1986). In particular, some of the DC models are based on the assumption that the magnetic and velocity fields of the coronal plasma are in a turbulent state (see for instance Gómez and Ferro Fontan, 1988, 1992; Heyvaerts and Priest, 1992; Einaudi et al., 1996; Hendrix and van Hoven, 1996) As a result of the existence of a strong, 
mean magnetic field, both the fluctuations and their turbulent wavenumbers are essentially two-dimensional, perpendicular to the mean field. Energy is transferred from photospheric motions to the magnetic field, cascaded to high perpendicular wavenumers and efficiently dissipated in highly structured current sheets.

It is clear from the discussion in previous sections that both open field-line and closed field-line models have a similar structure in what regard to the turbulent cascade and dissipation. The main difference lies in the nature of the driving, which in turn is related to the fundamental geometrical difference between these scenarios. While, in the absence of reflections, low frequency MHD fluctuations injected upwards from the photosphere would exit the corona without major dissipation in coronal holes, smaller loops contained in the corona can reflect injected waves back and forth at the photospheric boundary, thus naturally producing counter-propagating fluctuations, as needed to obtain turbulence. Moreover, fluctuations are constantly injected from both footpoints. In this regard, turbulence is harder to produce in open field-line regions. This may explain why closed loop models obtain reasonable heating rates even without considering reflections. Including reflections in these models might enhance dissipation, but more importantly may help describe quantities derived from vertical inhomogeneities, such as $H_{E}$ (Aschwanden et al., 2000). We note that even though our driving is wave-like, it includes quasi-static forcing as the particular case in which the frequency of the injected waves tends to zero. And it is particularly in the limit of low frequency where we find more efficient reflection. Further exploration of the relationship between these two distinct kinds of models may prove very useful, as what is learned in one context seems to be useful in the other.

\subsection{Anisotropic temperatures}

One of the most striking solar physics discoveries since the launch of SOHO has been the detection of anisotropically heated ions in coronal hole outflows. For those ions $T_{\perp}$ is much larger than $T_{\|}$(Kohl et al., 1998). Coronal proton temperatures are also observed to be several times higher than electron temperatures. Thus, a heating mechanism must supply energy directly to protons (and not, e.g. through electron heat conduction) and offset the tendency for protons moving though an expanding magnetic field to evolve towards higher parallel temperature. Combined with the short time available for heating, these circumstances have led to the suggestion that heating of the protons is due to direct cyclotron absorption of high-frequency Alfvén waves (McKenzie et al., 1995). While appealing in many respects, the cyclotron absorption scenario can be questioned on several points (Leamon et al., 2000). First, from an MHD perspective, the cascade most easily supplies energy to high perpendicular wavenumber, not to the high parallel wavenumbers that would be suggested by a cyclotron resonance condition. Second, if the wave flux is not resupplied, then a very "hard" spectrum of high-frequency waves is required to supply ade- quate energy to accelerate the wind; this has never been observed. Finally, Cranmer (2000) has pointed out that minor ions so effectively remove energy due to resonance with parallel propagating Alfvén waves that there may be insufficient power left to heat the protons (as they necessarily have resonance at higher frequency). Evidently some special kind of resupply of resonant waves is needed for the direct cyclotron absorption mechanism to work as a heating source for the major constituent of the corona, and at present that process stands as a mystery. An alternative picture would be welcome, provided that signatures such as high perpendicular proton temperature, often taken as a "smoking gun" of cyclotron resonance, can be explained.

So, how might we understand the occurrence of this anisotropy in the context of a cascade model? No detailed answer is yet available, but we suggest that some guidance may be found in recent studies of the Earth's aurora (Ergun et al., 1998). There, when electric fields occur aligned with the local magnetic field, the FAST spacecraft has detected the presence of 3D electron phase space holes travelling at the electron drift velocity. The structures have strong interactions with ions, leading specifically to substantial perpendicular heating of the ions (Carlson et al., 1998).

The heating process, as it is currently viewed in the auroral context, has some properties analogous to, if not similar to, the corona. This is not the first time that analogies between processes in the terrestrial aurora and processes in the sun have been pointed out (see, e.g. De Feiter, 1975). In each case there is a strong vertical magnetic field, and gravity that tends to confine the protons and ions more effectively than it does electrons. (In the aurora, gravitational effects will not be important when the proton temperatures exceed several ev.) The parallel electric field is a very important feature of both cases, but it is of different origin. In the lower corona, such parallel electric fields would be produced in patches throughout the region of strong MHD turbulence, as the transverse cascade processe terminates in small-scale transverse reconnection events (e.g. Matthaeus and Lamkin, 1986). The intermittent strong parallel electric field near these highly driven reconnection zones would be a prime candidate for very rapidly accelerating strong vertical electron beams. In their nonlinear stages, these would be expected to produce copious electron holes in analogy to the auroral context.

Suppose we assume that in the corona these nonlinear electron beams give rise to an extremely large number of vertically propagating charge structures moving at the electron thermal speed. Most likely their vertical dimension is of the order of the Debye scale, and their transverse scale is of the order of the electron gyroradius (Ergun et al., 1998). The rapid vertical propagation of these structures would produce a high-frequency electric field in the frame of the protons. If observations were feasible, there might be characteristic signatures such as those seen in the FAST electric field data (Ergun et al., 1998). Then protons, almost stationary at these time scales, would be bombarded with high-frequency uncorrelated transverse impulses that would increase their 
perpendicular kinetic energy. Protons in the corona would thus increase their $T_{\perp}$ until their parallel velocities increase (through pitch angle scattering or mirror force) to the gravitational escape speed $(600-700 \mathrm{~km} / \mathrm{s})$. In this view, in the corona the combination of gravity, reconnection, and electron beam dynamics might act as a "pressure cooker" that anisotropically heats the protons until they escape. An interesting feature of this scenario is that it might involve proton cyclotron motions, and excitation of associated cyclotron waves. However apart from the high perpendicular temperature, other "cyclotron signatures" are more or less incidental. We emphasize that the envisioned process is not a cyclotron resonant process in an essential way.

\section{Summary and discussion}

The mechanism by which the corona is heated and the solar wind accelerated is an important and enigmatic problem. It has defied solution for decades, as observations have provided ever more revealing and sometimes surprising features and constraints. Presently there are a number of efforts in differing directions to explain these processes, which are fundamental in solar, space and astrophysics. We have not attempted to be in any sense complete in our review of models currently being used to investigate coronal heating. Instead, we attempted to provide an observationally based overview, with the goal of motivating an anisotropic MHD turbulence-based model for open field-line coronal heating that our group has developed. We have been especially interested in defining the parameters of relevance to constraining such a model, which relies upon low-frequency fluctuations, transport, reflection from coronal inhomogeneities, and then a cascade process with terminates (in an MHD sense) with small-scale reconnection.

In our model MHD waves emitted by the solar photosphere are reflected in the overlying atmosphere. Much the same effects can be achieved by quasi-static driving, which we view as a limit of zero-frequency fluctuations supplied at the coronal base. Reflection from inhomogeneity of the Alfvén speed sets up counter-streaming waves, and this creates a cascade towards short perpendicular length-scales. Dissipation in small-scale reconnection events deposits energy in the medium. Using physical parameters that are plausible for the solar atmosphere, we find that the model leads to a quantitative understanding of at least part of the coronal heating problem.

A new feature of the present discussion is our conjecture concerning a possible dissipation mechanism that might operate near the turbulent reconnection zone driven by the cascade. Relying on high-frequency kinetic processes associated with electron phase space holes, strongly nonlinear kinetic couplings may be able to produce the high perpendicular proton temperatures indicated by recent coronal observations. This process may also accelerate and anisotropically heat minor ions, or it may leave the task of heating minor ions to the relatively less energetic parallel wave cascade (Lea- mon et al., 2000). By providing a completely separate channel for proton heating, this perpendicular heating process might be able to resolve the puzzles associated with models that rely solely upon direct cyclotron resonance (Cranmer, 2000) and parallel cascade. However it is clear that such ideas are preliminary and will require considerable work to evaluate. This is particularly difficult because the entire problem spans an enormous range of scales, involving driving and MHD timescales, and small-scale reconnection that triggers phenomena that are at electron time and length scales. On the other hand we suggest that efforts to examine this heating processes in the coronal context would benefit greatly from the large volume of observational and theoretical work currently being done on parallel electric fields, electron beams, and heating in the auroral context.

Acknowledgements. This research supported in part by NASA grant NAG5-8134 and NAG5-7164, NSF grants ATM-0105254 and ATM-9977692, and UK PPARC grant PPA/G/S/1999/00059.

We are particularly grateful to C. Carlson and G. Morales for useful discussion at the Troms $\varnothing$ meeting that led to the suggested analogies with auroral processes.

\section{References}

An, C.-H., Suess, S. T., Moore, R. L., and Musielak, Z. E.: Reflection and trapping of Alfvén waves in a spherically symmetric stellar atmosphere, Astrophys. J., 350, 309-323, 1990.

Aschwanden, M. J., Nightingale, R. W., and Alexander, D.: Evidence for Nonuniform Heating of Coronal Loops Inferred from Multithread Modeling of TRACE Data, Astrophys. J., 541, 1059-1077, 2000.

Axford, W. I. and McKenzie, J. F.: The solar wind, in: Cosmic Winds and the Heliosphere, Tucson, Univ. of Arizona Press, (Eds) Jokipii, J. R., Sonett, C. P., and Tiampapa, M. S., 31, 1997.

Barnes, A.: Hydromagnetic waves and turbulence in the solar wind, in: Solar System Plasma Physics, North-Holland Publishing Co., Amsterdam, 249-319, 1979.

Berger, T. E. and Title, A. M.: On the Dynamics of Small-Scale Solar Magnetic Elements, Astrophys. J., 463, 365, 1996.

Buchholz, B., Ulmschneider, P., and Cuntz, M.: Basal Heating in Main-Sequence Stars and Giants: Results from Monochromatic Acoustic Wave Models, Astrophys. J., 494, 700, 1998.

Carlson, C. W., McFadden, J. P., Ergun, R. E., Temerin, M., Peria, W., Mozer, F. S., Klumpar, D. M., Shelley, E. G., Peterson, W. K., Moebius, E., Elphic, R., Strangeway, R., Cattell, C., and Pfaff, R.: FAST observations in the downward auroral current region: Energetic upgoing electron beams, parallel potential drops, and ion heating, Geophys. Res. Lett. (GRL), 25, 2017, 1998.

Carlsson, M. and Stein, R. F.,: Formation of Solar Calcium H and K Bright Grains, Astrophys. J., 481, 500, 1997.

Chae, J., Schühle, U., and Lemaire, P.: SUMER Measurements of Nonthermal Motions: Constraints on Coronal Heating Mechanisms, Astrophys. J., 505, 957-973, 1998.

Cranmer, S. R.: Ion Cyclotron Wave Dissipation in the Solar Corona: The Summed Effect of More than 2000 Ion Species, Astrophys. J., 532, 1197-1208, 2000.

de Feiter, L. D.: Chromospheric flares or chromospheric aurorae, Space Science Reviews, 17, 181-193, 1975. 
Dmitruk, P., Matthaeus, W. H., Milano, L. J., and Oughton, S.: Conditions for sustainment of magnetohydrodynamic turbulence driven by Alfvén waves, Physics of Plasmas, 8, 2377-2384, 2001.

Einaudi, G., Velli, M., Politano, H., and Pouquet, A.: Energy Release in a Turbulent Corona, Astrophys. J. Lett., 457, L113, 1996.

Ergun, R. E., Carlson, C. W., McFadden, J. P., Mozer, F. S., Muschietti, L., Roth, I., and Strangeway, R. J.: Debye-Scale Plasma Structures Associated with Magnetic-Field-Aligned Electric Fields, Physical Review Letters, 81, 826-829, 1998.

Foukal, P.: Solar astrophysics, New York, Wiley-Interscience, 492, 1990.

Gomez, D. and Ferro Fontan, C.: Coronal heating by selective decay of MHD turbulence, Solar Phys., 116, 33-44, 1988.

Gomez, D. O. and Ferro Fontan, C.: Development of magnetohydrodynamic turbulence in coronal loops, Astrophys. J., 394, 662669, 1992.

Grall, R. R., Coles, W. A., Klinglesmith, M. T., Breen, A. R., Williams, P. J. S., Markkanen, J., and Esser, R.: Rapid acceleration of the polar solar wind, Nature, 379, 429-432, 1996.

Hammer, R. and Ulmschneider, P.: On the intrinsic difficulty of producing stellar coronae with acoustic waves, in Mechanisms of Chromospheric and Coronal Heating, Berlin, Springer Verlag, 344, 1991.

Hassler, D. M., Rottman, G. J., Shoub, E. C., and Holzer, T. E.: Line broadening of MG X 609 and 625 A coronal emission lines observed above the solar limb, Astrophys. J. Lett., 348, L77L80, 1990.

Hendrix, D. L. and van Hoven, G.: Magnetohydrodynamic Turbulence and Implications for Solar Coronal Heating, Astrophys. J., 467, 887, 1996.

Heyvaerts, J. and Priest, E. R.: Coronal heating by phase-mixed shear Alfvén waves, Astron. Astrophys., 117, 220-234, 1983.

Ionson, J. A.: A unified theory of electrodynamic coupling in coronal magnetic loops - The coronal heating problem, Astrophys. J., 276, 357-368, 1984.

Kohl, J. L., Gardner, L. D., Strachan, L., Fisher, R., and Guhathakurta, M.: SPARTAN 201 Coronal Spectroscopy During the Polar Passes of ULYSSES, Space Science Reviews, 72, 29, 1995.

Kohl, J. L., Noci, G., Antonucci, E., Tondello, G., Huber, M. C. E., Gardner, L. D., Nicolosi, P., Strachan, L., Fineschi, S., Raymond, J. C., Romoli, M., Spadaro, D., Panasyuk, A., Siegmund, O. H. W., Benna, C., Ciaravella, A., Cranmer, S. R., Giordano, S., Karovska, M., Martin, R., Michels, J., Modigliani, A., Naletto, G., Pernechele, C., Poletto, G., and Smith, P. L.: First Results from the SOHO Ultraviolet Coronagraph Spectrometer, Solar Phys., 175, 613-644, 1997.

Kohl, J. L., Noci, G., Antonucci, E., Tondello, G., Huber, M. C. E., Cranmer, S. R., Strachan, L., Panasyuk, A. V., Gardner, L. D., Romoli, M., Fineschi, S., Dobrzycka, D., Raymond, J. C., Nicolosi, P., Siegmund, O. H. W., Spadaro, D., Benna, C., Ciaravella, A., Giordano, S., Habbal, S. R., Karovska, M., Li, X., Martin, R., Michels, J. G., Modigliani, A., Naletto, G., O’Neal, R. H., Pernechele, C., Poletto, G., Smith, P. L., and Suleiman, R. M.: UVCS/SOHO Empirical Determinations of Anisotropic Velocity Distributions in the Solar Corona, Astrophys. J. Lett., 501, L127, 1998.
Leamon, R. J., Matthaeus, W. H., Smith, C. W., Zank, G. P., Mullan, D. J., and Oughton, S.: MHD-driven Kinetic Dissipation in the Solar Wind and Corona, Astrophys. J., 537, 1054-1062, 2000.

Leer, E., Holzer, T. E., and Fla, T.: Acceleration of the solar wind, Space Science Reviews, 33, 161-200, 1982.

Matthaeus, W. H. and Lamkin, S. L.: Turbulent magnetic reconnection, Physics of Fluids, 29, 2513-2534, 1986.

Matthaeus, W. H., Zank, G. P., Oughton, S., Mullan, D. J., and Dmitruk, P.: Coronal Heating by Magnetohydrodynamic Turbulence Driven by Reflected Low-Frequency Waves, Astrophys. J. Lett., 523, L93-L96, 1999.

McKenzie, J. F., Banaszkiewicz, M., and Axford, W. I.: Acceleration of the high speed solar wind., Astron. Astrophys., 303, L45, 1995.

Milano, L. J., Gomez, D. O., and Martens, P. C. H.: Solar Coronal Heating: AC versus DC, Astrophys. J., 490, 442-451, 1997.

Mullan, D. J. and Cheng, Q. Q., MG II and Ly-alpha fluxes in M dwarfs - Evaluation of an acoustic model, Astrophys. J., 412, 312-323, 1993.

Mullan, D. J. and Cheng, Q. Q.: Acoustic waves in M dwarfs: Maintaining a corona, Astrophys. J., 420, 392-400, 1994.

Mullan, D. J. and Fleming, T. A.: Coronal Heating in dMe and dM Stars: Clues from the X-Ray Surface Fluxes, Astrophys. J., 464, 890, 1996.

Musielak, Z. E., Rosner, R., Stein, R. F., and Ulmschneider, P.: On sound generation by turbulent convection: A new look at old results, Astrophys. J., 423, 474-487, 1994.

Namba, O. and Diemel, W. E.: A Morphological Study of the Solar Granulation, Solar Phys., 7, 167, 1969.

Oughton, S., Matthaeus, W. H., Dmitruk, P., Milano, L. J., Zank, G. P., and Mullan, D. J.: A Reduced Magnetohydrodynamic Model of Coronal Heating in Open Magnetic Regions Driven by Reflected Low-Frequency Alfvén Waves, Astrophys. J., 551, 565-575, 2001.

Parker, E. N.: Topological Dissipation and the Small-Scale Fields in Turbulent Gases, Astrophys. J., 174, 499, 1972.

Parker, E. N.: Magnetic Neutral Sheets in Evolving Fields - Part Two - Formation of the Solar Corona, Astrophys. J., 264, 642, 1983.

Parker, E. N.: Nanoflares and the solar X-ray corona, Astrophys. J., 330, 474-479, 1988.

Parker, E. N.: Vortex attraction and the formation of sunspots, Astrophys. J., 390, 290-296, 1992.

Schrijver, C. J. and Aschwanden, M. J.: Constraining the Properties of Nonradiative Heating of the Coronae of Cool Stars and the Sun, Astrophys. J., 566, 1147-1165, 2002.

Stein, R. F. and Schwartz, R. A.: Waves in the Solar Atmosphere. III. The Propagation of Periodic Wave Trains in a Gravitational Atmosphere, Astrophys. J., 186, 1083-1090, 1973.

Ulmschneider, P. (Ed.): Acoustic heating, 1991.

van Ballegooijen, A. A.: Cascade of magnetic energy as a mechanism of coronal heating, Astrophys. J., 311, 1001-1014, 1986.

Vernazza, J. E., Avrett, E. H., and Loeser, R.: Structure of the solar chromosphere. III - Models of the EUV brightness components of the quiet-sun, APJ.S., 45, 635-725, 1981.

Withbroe, G. L. and Noyes, R. W.: Mass and energy flow in the solar chromosphere and corona, ARA \& A, 15, 363-387, 1977. 